\title{
Detox diet-worth to use it or not?
}

\begin{abstract}
Recent global statistics reveal a rapid increase in obesity amongst all socioeconomic and age groups globally. People nowadays have adapted sedentary lifestyle pattern which is one of the main cause behind this epidemic. Most of the population is in search of quick and easy ways of losing weight, many quick fix products, formulas and diets are being introduced and are gaining public interest especially by the youth. One of these quick fix methods is detoxification or detox diets. This dietary pattern revolves mostly around consumption of fruits, vegetables and water. Lack of major nutrients like proteins, fats and essential vitamins and minerals may lead to many deficiencies. Adapting the dietary pattern may also lead to loss of minerals and water due to the laxative regimen. The effectiveness of such diets is not even scientifically proven. A balanced diet and regular physical activity can help you fulfill your aim of leading a healthy life without risking your health.
\end{abstract}

Keywords: obesity, detoxification, detox, laxatives, diet, toxins, toxicity
Opinion

Volume 8 Issue 2 - 2018

\author{
Syeda Tooba Ali \\ Clinical Dietitian, Aga Khan University Hospital, Pakistan
}

Correspondence: Syeda Tooba Ali, Clinical Dietitian/RD, Aga Khan University Hospital, Pakistan, Email alitooba22@gmail.com

Received: December 22, 2017 | Published: March 01, 2018
Abbreviations: WHO, world health organization; KCAL, kilocalories; FDA, food and drug administration.

\section{Opinion}

Obesity is an upcoming health concern which is now turning into an epidemic. As per WHO's 2016 statistics more than 1.9 billion adults fall into the category of overweight of which 650 million came out to be obese. Obesity is a complex condition which causes an impact over all age groups as well as socioeconomic statuses not only in developed countries but in developing countries as well. Obesity brings serious health complexities with it such as Diabetes, Cardiovascular diseases, dyslipidemia, Osteoarthritis, Stroke, Gall bladder and liver diseases, breathing problems like sleep apnea, also some psychological disorders like anxiety and depression. One of the major reasons behind this increasing obesity pattern is sedentary lifestyle, as said sitting is the new smoking. We rely for most our needs on technology and luxuries for fast and effective results. By adapting this robotic pattern of life, we're welcoming many health complications.

With an increase in obesity statistics, people have started inventing easy and quick ways to reduce weight and one of the new topics of talk in town is 'detox', a trend which is being followed by many people specially youth. Detox comes from detoxification which means removing toxic substances from your body. The word "detox" is referred chiefly to a medical procedure that rids the body of dangerous, often life-threatening, levels of alcohol, drugs, or poisons. The treatment is based mostly on the use of drugs and different therapies combined, depending on the kind and level of the toxicity. Toxins, chemical or any poisonous substance can come from food that we eat and the air that we breathe and to get rid of these harmful substances nature has designed our bodies to process those toxins through different organs like kidneys as well as the liver and these organs function by eliminating them in the form of waste products in the form of urine, feces and perspiration. The detox diet theories are not proven scientifically. Extremely low-calorie diets invariably lower the body's basal metabolic rate as it struggles to conserve energy.
Once normal eating patterns are resumed by the dieter, rapid weight gain follows. Much of the weight loss achieved through this diet results from fluid loss related to extremely low carbohydrate intake and frequent bowel movements or diarrhea produced by salt water and laxative tea. When the dieter resumes normal fluid intake, this weight is quickly regained.

The detox diets encourage you to eat natural foods including lots of veggies and massive amounts of water. People specially youth gets more attracted towards it after seeing their favorite showbiz celebrities following these diets with heavy gym workouts. These diets do give us results by rapidly reducing our weight within a small duration of time. But this is not stupendous because calorie intakes are usually extremely low and lack major groups of foods such as milk and its products, meat and crabs in the form of wheat-based foods means you'll slash the amount of calories you have - and only replace a few of these calories with the extra fruit and vegetables, beans, seeds, nuts and herbal teas. Detox diets may be helpful in losing weight but the adaptor of such diets should be cautious about the adverse effects of it. This dietary pattern lacks in protein, fatty acids, and other essential vitamins and minerals. Carbohydrates supply all the calories-an extremely low approx. $600 \mathrm{kcal}$. The daily laxative regimen can cause dehydration, deplete electrolytes, and impair normal bowel function. It can also disrupt the native intestinal flora, microorganisms that perform useful digestive functions. A person who goes on this diet repeatedly may run the risk of developing metabolic acidosis, a disruption of the body's acid-base balance, which results in excessive acidity in the blood. Severe metabolic acidosis can lead to coma and death. People who fast for several days may drop pounds, but most of it will be water and some of it may be muscle. These diets doesn't burn body's fat.

These detox diets are contra indicated for patients with diseases like Diabetes, Cardiovascular diseases or any chronic diseases, pregnancy and at that period of life when youth especially teens are going through a phase of rapid growth and development where there nutritional needs are at a risk of being highly compromised. Apart from these do-it-yourself methods to eliminate alleged toxins that are held 
responsible for variety of health related issues, many detox products are being marketed as "whole body cleansers" or advertised as an instant tool of getting smart at retail stores, at spas, over the internet or by direct mail. These products are not available by prescription of any health professionals. These kinds of supplements contain laxative properties, which increase your gut motility and make you poop very often throughout the day and that can get messy. Laxatives are not a great idea because they can lead to dehydration, gastrointestinal discomforts and mineral loss. The U.S. Food and Drug Administration (FDA) and Federal Trade Commission have taken action against several companies selling detox/cleansing products because they contained illegal, potentially harmful ingredients; were marketed using false claims. ${ }^{1-3}$

Being concerned about your weight and to have a desire for living a healthier life is not something wrong but adapting dietary regimens of eating fruits and vegetables and drinking plenty of water only is not advisable as by omitting fat, proteins and compromising vitamins and minerals from diet puts you on risk of many nutritional deficiencies.
The human body has a well-designed mechanism to guard itself from environmental insults. A well-balanced diet containing adequate amounts of proteins, fat, carbohydrates, vitamins and minerals with adequate fluid intake, regular physical activity and sufficient sleep is the key to a person's healthy body and a peaceful mind. Because a healthy life is a happy life!

\section{Acknowledgement}

None.

\section{Conflicts of interest}

There are no conflicts of interest.

\section{References}

1. The dubious practice of detox.

2. What Is a Detox Diet?

3. "Detoxes" and "Cleanses" 